\title{
MORDELL EXCEPTIONAL LOCUS FOR SUBVARIETIES OF THE ADDITIVE GROUP
}

\author{
Dragos GHIOCA
}

\begin{abstract}
We define the Mordell exceptional locus $Z(V)$ for affine varieties $V \subset \mathbb{G}_{a}^{g}$ with respect to the action of a product of Drinfeld modules on the coordinates of $\mathbb{G}_{a}^{g}$. We show that $Z(V)$ is a closed subset of $V$. We also show that there are finitely many maximal algebraic $\phi$-modules whose translates lie in $V$. Our results are motivated by Denis-Mordell-Lang conjecture for Drinfeld modules.
\end{abstract}

\section{Introduction}

Faltings proved the Mordell-Lang conjecture in the following form (see [Fal94]).

Theorem 1.1 (Faltings). Let $G$ be an abelian variety defined over the field of complex numbers $\mathbb{C}$. Let $X \subset G$ be a closed subvariety and $\Gamma \subset G(\mathbb{C})$ a finitely generated subgroup of $G(\mathbb{C})$. Then $X(\mathbb{C}) \cap \Gamma$ is a finite union of cosets of subgroups of $\Gamma$.

In particular, Theorem 1.1 says that an irreducible subvariety $X$ of an abelian variety $G$ has a Zariski dense intersection with a finitely generated subgroup of $G(\mathbb{C})$ only if $X$ is a translate of an algebraic subgroup of $G$.

We define the Mordell exceptional locus of $X \subset G$ as the set (see also [Abr94])

$$
Z(X)=\{x \in X \mid \exists B, \operatorname{dim} B>0, B \text { an algebraic subgroup, } x+B \subset X\} .
$$

Thus, Theorem 1.1 says that for each finitely generated subgroup $\Gamma$, we have that $(X \backslash Z(X)) \cap \Gamma$ is finite. The Mordell exceptional locus of subvarieties of abelian varieties was shown to be closed (see [Kaw80], [Bog81] and [Abr94]). This last paper served as inspiration for our work.

If we try to formulate the Mordell-Lang conjecture in the context of algebraic subvarieties contained in a power of the additive group scheme $\mathbb{G}_{a}$, the conclusion is either false (in the characteristic 0 case, as shown by the curve $y=x^{2}$ which has an infinite intersection with the finitely generated subgroup $\mathbb{Z} \times \mathbb{Z}$, without being itself a translate of an algebraic subgroup of $\mathbb{G}_{a}^{2}$ ) or it is trivially true (in the characteristic $p>0$ case, because every finitely generated subgroup of a power of $\mathbb{G}_{a}$ is finite). Denis [Den92] formulated a Mordell-Lang conjecture for powers of $\mathbb{G}_{a}$ in characteristic $p$ in the context of Drinfeld modules. Denis replaced the finitely generated subgroup from the usual Mordell-Lang statement with a finitely generated $\phi$-submodule, where $\phi$ is a Drinfeld module. He also strengthened the conclusion of the Mordell-Lang statement by asking that the subgroups whose cosets are contained in the intersection of the algebraic variety with the finitely generated $\phi$-submodule be actually $\phi$-submodules.

Received by the editors January 29, 2007.

2000 Mathematics Subject Classification. Primary 11G09, Secondary 14L10, 14K12.

Key words and phrases. Drinfeld module, Mordell exceptional locus. 
Several cases of Denis-Mordell-Lang conjecture were proved by the author (see [Ghi05] and [Ghi06]), and by Thomas Tucker and the author (see [GT07]).

Similar with the case of abelian varieties, Denis-Mordell-Lang conjecture suggests that the intersection of a variety $V$ with a finitely generated $\phi$-module should be finite outside the Mordell exceptional locus $Z(V)$ of $V$ (see our Definition 2.2 for $Z(V)$ ). In the present paper we prove that $Z(V)$ is closed (see our Theorem 2.4). In addition, we show that there exists no infinite family of algebraic $\phi$-modules (see Corollary 3.9 ), and that for every affine variety $V \subset \mathbb{G}_{a}^{g}$ there are finitely many maximal algebraic $\phi$-modules whose translates lie in $V$ (see our Theorem 2.5). Both of these statements are further indications that Denis-Mordell-Lang conjecture should be true.

We briefly sketch the plan of our paper. In Section 2 we set the notation, describe the Denis-Mordell-Lang conjecture and then state our main results. In Section 3 we prove these main results (Theorems 2.4 and 2.5).

\section{Notation and statement of our main results}

All subvarieties appearing in this paper are closed. We define next the notion of a Drinfeld module.

Let $p$ be a prime and let $q$ be a power of $p$. Let $C$ be a projective non-singular curve defined over $\mathbb{F}_{q}$. Let $A$ be the ring of $\mathbb{F}_{q}$-valued functions defined on $C$, regular away from a fixed closed point $\infty \in C$. Let $K$ be a finite field extension of the fraction field $\operatorname{Frac}(A)$ of $A$. We let $K^{\text {alg }}$ be a fixed algebraic closure of $K$, and let $K^{\text {sep }}$ be the separable closure of $K$ inside $K^{\text {alg }}$.

We define the operator $\tau$ as the Frobenius on $\mathbb{F}_{q}$, extended so that for every $x \in$ $K^{\text {alg }}$, we have $\tau(x)=x^{q}$. Then for every subfield $L \subset K^{\text {alg }}$, we let $L\{\tau\}$ be the ring of polynomials in $\tau$ with coefficients from $L$ (the addition is the usual addition, while the multiplication is given by the usual composition of functions).

Following Goss [Gos96], we call a Drinfeld module of generic characteristic defined over $K$ a morphism $\phi: A \rightarrow K\{\tau\}$ for which the coefficient of $\tau^{0}$ in $\phi_{a}$ is $a$ for every $a \in A$, and there exists $a \in A$ such that $\phi_{a} \neq a \tau^{0}$. All Drinfeld modules appearing in this paper are of generic characteristic.

For every field extension $K \subset L$, any Drinfeld module $\phi$ induces an action on $\mathbb{G}_{a}(L)$ by $a * x:=\phi_{a}(x)$, for each $a \in A$.

Let $g$ be a fixed positive integer. Let $\phi_{1}: A \rightarrow K\{\tau\}, \ldots, \phi_{g}: A \rightarrow K\{\tau\}$ be Drinfeld modules. From now on, we denote by $\phi$ the $\left(\phi_{1}, \ldots, \phi_{g}\right)$-action on $\mathbb{G}_{a}^{g}$ (where each Drinfeld module $\phi_{i}$ acts on the corresponding coordinate of the affine space).

A point $x \in \mathbb{G}_{a}^{g}\left(K^{\text {alg }}\right)$ is torsion for the $\phi$-action if there exists $a \in A \backslash\{0\}$ such that $\phi_{a}(x)=0$. We denote by $\phi[a]$ the finite set of all torsion points $x$ killed by $\phi_{a}$. We denote by $\phi_{\text {tor }}$ the set of all torsion points in $\mathbb{G}_{a}^{g}\left(K^{\text {alg }}\right)$.

The subgroups of $\mathbb{G}_{a}^{g}\left(K^{\mathrm{alg}}\right)$ invariant under the action of $\phi$ are called $\phi$-submodules.

Definition 2.1. An algebraic $\phi$-(sub)module of $\mathbb{G}_{a}^{g}$ is an irreducible algebraic subgroup of $\mathbb{G}_{a}^{g}$ invariant under $\phi$.

Now we can define the Mordell exceptional locus of an affine subvariety $V \subset \mathbb{G}_{a}^{g}$.

Definition 2.2. Let $V \subset \mathbb{G}_{a}^{g}$ be an affine subvariety. We let $Z(V)$ be the set of all points $y \in V$ with the property that there exists a positive dimensional algebraic $\phi$-submodule $Y \subset \mathbb{G}_{a}^{g}$ such that $(y+Y) \subset V$. 
Denis proposed in [Den92] the following conjecture.

Conjecture 2.3. Let $V \subset \mathbb{G}_{a}^{g}$ be an affine variety defined over $\bar{K}$. Let $\Gamma$ be a finitely generated $\phi$-submodule of $\mathbb{G}_{a}^{g}(\bar{K})$. Then there exist algebraic $\phi$-submodules $B_{1}, \ldots, B_{l}$ of $\mathbb{G}_{a}^{g}$ and there exist $\gamma_{1}, \ldots, \gamma_{l} \in \Gamma$ such that

$$
V(\bar{K}) \cap \Gamma=\bigcup_{i=1}^{l}\left(\gamma_{i}+B_{i}(\bar{K})\right) \cap \Gamma .
$$

As explained in Introduction, the results of our paper were motivated by Conjecture 2.3. Our main result is the following.

Theorem 2.4. With the above notation for $\phi$ and $V$, the Mordell exceptional locus $Z(V)$ is Zariski closed.

The following important result is a consequence of Theorem 2.4.

Theorem 2.5. Let $V \subset \mathbb{G}_{a}^{g}$ be an affine subvariety. There are finitely many maximal algebraic $\phi$-submodules $Y$ such that a translate of $Y$ lies in $V$ (where $Y$ is maximal in the sense that there is no larger algebraic $\phi$-module whose translate lies in $V$ ).

\section{Proofs of our main results}

We continue with the notation from Section 2. Hence $\phi_{1}, \ldots, \phi_{g}$ are Drinfeld modules, and we denote by $\phi$ the action of $\left(\phi_{1}, \ldots, \phi_{g}\right)$ on $\mathbb{G}_{a}^{g}$. Unless otherwise stated, $V \subset \mathbb{G}_{a}^{g}$ is an affine subvariety, and $Z(V)$ is its Mordell exceptional locus (as defined in Definition 2.2).

We first state a result which we will use later (Lemme 4 of [Den92]).

Lemma 3.1 (Denis). Let $Y \subset \mathbb{G}_{a}^{g}$ be an irreducible subvariety, and let $t \in A$ be a non-constant function. If $\phi_{t}(Y)=Y$, then $Y$ is a translate of an algebraic $\phi$-module.

The following corollary follows easily from Lemma 3.1.

Corollary 3.2. Let $0 \in Y \subset \mathbb{G}_{a}^{g}$ be an irreducible subvariety, and let $t \in A$ be a non-constant function. If $\phi_{t}(Y)=Y$, then $Y$ is an algebraic $\phi$-module.

Proof. According to Lemma 3.1, $Y=y+Z$ is a translate of an algebraic $\phi$-module $Z$. Because $0 \in Y$, then $-y \in Z$, and so, because $Z$ is an algebraic group, we conclude that $y+Z=Z$. Therefore $Y=Z$ is an algebraic $\phi$-module.

The following Fact is a consequence of Lemma 3.1.

Fact 3.3. Let $t \in A$ be a non-constant function. Let $0 \in Y \subset \mathbb{G}_{a}^{g}$ be a variety such that $\phi_{t}(Y) \subset Y$. Let $Z$ be an irreducible component of $Y$ containing 0 . Then $Z$ is an algebraic $\phi$-module.

Proof. First we prove the following Claim.

Claim 3.4. Let $Z$ be an irreducible subvariety of $\mathbb{G}_{a}^{g}$ containing 0 . Suppose that for some positive integers $m<n$, we have $\phi_{t^{m}}(Z)=\phi_{t^{n}}(Z)$. Then $Z$ is an algebraic $\phi$-module. 
Proof of Claim 3.4. By our assumption, the irreducible subvariety $\phi_{t^{m}}(Z)$ is invariant under $\phi_{t^{n-m}}$. Hence, using Corollary 3.2, we conclude that $Z_{0}:=\phi_{t^{m}}(Z)$ is an algebraic $\phi$-module. In particular, $\phi_{t^{m}}\left(Z_{0}\right)=Z_{0}$. Thus, using that $\phi_{t^{m}}(Z)=\phi_{t^{m}}\left(Z_{0}\right)$, we get that

$$
Z \subset \bigcup_{z \in \phi\left[t^{m}\right]}\left(z+Z_{0}\right)
$$

Because $Z$ is irreducible, then (3.4.1) yields that there exists $z \in \phi\left[t^{m}\right]$ such that $Z \subset$ $\left(z+Z_{0}\right)$. Because $\operatorname{dim}(Z)=\operatorname{dim}\left(\phi_{t^{m}}(Z)\right)=\operatorname{dim}\left(Z_{0}\right)$, and because $Z_{0}$ is irreducible, we conclude that $Z=z+Z_{0}$. Because $0 \in Z=z+Z_{0}$, we obtain that $-z \in Z_{0}$, and so, $Z=z+Z_{0}=Z_{0}$ is an algebraic $\phi$-module, as desired.

The following result is an easy corollary of Claim 3.4.

Corollary 3.5. Let $\ell$ be a positive integer, and let $S:=\left\{Y_{i}\right\}_{i=1}^{\ell}$ be a finite set of irreducible subvarieties of $\mathbb{G}_{a}^{g}$, each containing 0 , such that $\phi_{t}$ acts on $S$ (by permuting the varieties). Then each $Y_{i}$ is an algebraic $\phi$-module.

Proof of Corollary 3.5. Because $\phi_{t}$ acts on the finite set $S$, then there exist positive integers $m<n$ such that for each $i \in\{1, \ldots, \ell\}$, we have $\phi_{t^{m}}\left(Y_{i}\right)=\phi_{t^{n}}\left(Y_{i}\right)$. Then Claim 3.4 yields the conclusion of Corollary 3.5.

Let $d$ be the maximal dimension of the irreducible components of $Y$ passing through 0 . Let $Z$ be an irreducible component of $Y$, passing through 0 . We will prove Fact 3.3 by induction on $s:=d-\operatorname{dim}(Z)$.

First we prove the case $s=0$. So, let $\left\{Z_{i}\right\}_{i=1}^{\ell}$ be all the irreducible components of $Y$ of dimension $d$, which contain 0 . Because $0 \in \phi_{t}\left(Z_{i}\right) \subset \phi_{t}(Y) \subset Y$, then $\phi_{t}\left(Z_{i}\right)$ is contained in an irreducible component $Z_{j}$ of $Y$ (of maximal dimension $d$, because $\left.\operatorname{dim}\left(\phi_{t}\left(Z_{i}\right)\right)=\operatorname{dim}\left(Z_{i}\right)=d\right)$, which passes through 0 . Because $\operatorname{dim}\left(\phi_{t}\left(Z_{i}\right)\right)=$ $\operatorname{dim}\left(Z_{j}\right)$ and both $\phi_{t}\left(Z_{i}\right)$ and $Z_{j}$ are irreducible, we conclude that $\phi_{t}\left(Z_{i}\right)=Z_{j}$. Hence, $\phi_{t}$ acts on the finite set $\left\{Z_{i}\right\}_{i=1}^{\ell}$. Thus, Corollary 3.5 yields that each $Z_{i}$ is an algebraic $\phi$-module.

Let $s \geq 1$. We assume that we proved Fact 3.3 for all irreducible components of dimension greater than $(d-s)$, and we will prove next that Fact 3.3 holds also for the irreducible components of dimension $d-s$.

Let $T:=\left\{W_{i}\right\}_{i=1}^{k}$ be all the irreducible components of $Y$ of dimension $(d-s)$, which contain 0. If $\phi_{t}$ acts on the finite set $T$, then Corollary 3.5 yields that each $W_{i}$ is an algebraic $\phi$-module, as desired. Therefore, assume from now on that $\phi_{t}$ does not act on $T$. However, for each $W:=W_{i}$, there exists another irreducible component $Z$ of $Y$ passing through 0 , such that $\phi_{t}(W) \subset Z$. Assume $Z \notin T$. Then $\operatorname{dim}(Z)>d-s$. By the induction hypothesis, $Z$ is an algebraic $\phi$-module. Hence, because $Z=\phi_{t}(Z)$ contains $\phi_{t}(W)$, then

$$
W \subset \bigcup_{y \in \phi[t]} y+Z
$$

Because $W$ is irreducible, then there exists $y \in \phi[t]$ such that $W \subset y+Z$. But $0 \in W$, and so, $0 \in y+Z$. Therefore $-y \in Z$, and because $Z$ is an algebraic group, we conclude that $y+Z=Z$. Hence $W \subset Z$, which contradicts the fact that $W$ is an 
irreducible component of $Y$, different from $Z$. This contradiction shows that actually $\phi_{t}$ acts on the finite set $T$, and so, it concludes our inductive proof.

We are ready to prove that $Z(V)$ is a (closed) subvariety of $V$.

Proof of Theorem 2.4. Our proof follows the second proof of Theorem 1 from [Abr94]. Let $t \in A$ be a non-constant function.

For each $m \geq 2$ we define the map $F_{m}:\left(\mathbb{G}_{a}^{g}\right)^{m} \rightarrow\left(\mathbb{G}_{a}^{g}\right)^{m-1}$ by

$$
\left(y_{1}, \ldots, y_{m}\right) \rightarrow\left(\phi_{t}\left(y_{1}\right)-y_{2}, \phi_{t}\left(y_{2}\right)-y_{3}, \ldots, \phi_{t}\left(y_{m-1}\right)-y_{m}\right) .
$$

Clearly, the map $F_{m}^{\prime}: \mathbb{G}_{a}^{g m} \rightarrow \mathbb{G}_{a}^{g m}$ given by

$$
F_{m}^{\prime}\left(y_{1}, \ldots, y_{m}\right):=\left(y_{1}, \phi_{t}\left(y_{1}\right)-y_{2}, \phi_{t}\left(y_{2}\right)-y_{3}, \ldots, \phi_{t}\left(y_{m-1}\right)-y_{m}\right)
$$

is an isomorphism. We let $F_{m}^{V}$ be the map $F_{m}$ restricted to $V^{m}$.

We let $D_{m}: V \rightarrow \mathbb{G}_{a}^{g(m-1)}$ defined by $D_{m}(y)=\phi_{t-1} \cdot(y, y, \ldots, y)$. We let $Y_{m} \subset$ $V^{m+1}$ be defined as

$$
\left\{\left(y_{1}, \ldots, y_{m}, y\right) \in V^{m} \times V \mid F_{m}^{V}\left(y_{1}, \ldots, y_{m}\right)=D_{m}(y)\right\} .
$$

Using the fact that $F_{m}^{\prime}$ is an isomorphism, we obtain that $Y_{m}$ embeds into $V \times V$ via the map

$$
\left(y_{1}, \ldots, y_{m}, y\right) \rightarrow\left(y_{1}, y\right) .
$$

Let $Y_{m}^{\prime} \subset V \times V$ be the image of $Y_{m}$ through the map in (3.5.3). We claim that for $n>m$, we have $Y_{n}^{\prime} \subset Y_{m}^{\prime}$.

Indeed, if $\left(y_{1}, \ldots, y_{n}, y\right) \in Y_{n}$, then $\left(y_{1}, \ldots, y_{m}, y\right) \in Y_{m}$. Therefore $\left\{Y_{m}^{\prime}\right\}_{m \geq 2}$ is a descending chain of subvarieties of $V \times V$, which has to stabilize. Hence, for some positive integer $n$, we have $Y_{m}^{\prime}=Y_{n}^{\prime}$ for each $m \geq n$.

We note that each $Y_{m}$ contains the diagonal of $V^{m+1}$. Hence, each $Y_{m}^{\prime}$ contains the diagonal $\Delta$ of $V \times V$.

We have the natural projection $\pi_{2}$ of $Y_{n}^{\prime} \subset V \times V$ on the second coordinate. The following Claim is the key to our proof.

Claim 3.6. For each $y \in V$, and for each irreducible component $Z \times\{y\}$ of the fiber $\pi_{2}^{-1}(y)$, which passes through $(y, y)$, the translate $-y+Z$ is an algebraic $\phi$ module. Moreover, $\pi_{2}^{-1}(y)$ contains a positive dimensional irreducible component passing through $(y, y)$ if and only if there exists a positive dimensional algebraic $\phi$ module $Z$ such that $(y+Z) \subset V$, if and only if $y \in Z(V)$.

Proof of Claim 3.6. Let $y \in V$, and let $Y^{\prime} \times\{y\}=\pi_{2}^{-1}(y) \subset Y_{n}^{\prime}$ be the fiber above $y$. Hence $Y^{\prime} \subset V$, and we let $Y:=\left(Y^{\prime}-y\right)$. Then $0 \in Y$ (because $\Delta \subset Y_{n}^{\prime}$, and so, $\left.y \in Y^{\prime}\right)$. We claim that $\phi_{t}(Y) \subset Y$.

Indeed, every point $y_{1} \in Y^{\prime}$ lies below a point $\left(y_{1}, y\right) \in Y_{n}^{\prime}$, and in addition because $\left\{Y_{m}^{\prime}\right\}_{m}$ stabilizes for $m \geq n$, we obtain that $\left(y_{1}, y\right) \in Y_{m}^{\prime}$ for all $m \geq n$. In particular, using (3.5.2), we conclude that there exists an infinite sequence $\left\{y_{i}\right\}_{i \geq 1} \subset V$ such that

$$
\phi_{t}\left(y_{i}\right)-y_{i+1}=\phi_{t-1}(y) \text { for every } i \geq 1 \text {. }
$$

Therefore,

$$
\phi_{t}\left(y_{i}-y\right)=y_{i+1}-y \text { for all } i \geq 1 \text {. }
$$


Moreover, (3.6.1) yields that also $\left(y_{2}, y\right) \in Y_{n}^{\prime}$, and so $\left(y_{2}-y\right) \in Y$. Hence, (3.6.2) for $i=1$ yields that $\phi_{t}(Y) \subset Y$. Therefore, using Fact 3.3, we conclude that each irreducible component of $Y$ containing 0 is an algebraic $\phi$-module.

Now, conversely, assume $(y+Y) \subset V$ and $Y$ is maximal in the sense that there exists no larger algebraic $\phi$-module whose translate by $y$ lies in $V$. Then, because $Y$ is invariant under $\phi_{t}$, for each $y_{1} \in(y+Y)$ there exists an infinite sequence

$$
\left\{y_{i}\right\}_{i \geq 1} \subset(y+Y) \subset V
$$

such that (3.6.2) holds, and so, (3.6.1) holds. Therefore $\left(y_{1}, y\right) \in Y_{n}^{\prime}$, and so, $(y+$ $Y) \times\{y\}$ lies in an irreducible component $Z \times\{y\}$ of the fiber $\pi_{2}^{-1}(y)$. We note that $Z \times\{y\}$ passes through $(y, y)$ because $y \in(y+Y)$. Moreover, as shown in the above paragraph, $Z \subset V$ is a translate by $y$ of an algebraic $\phi$-module. Because $Y$ is maximal, then $(y+Y)=Z$. Hence $\operatorname{dim}(Z)>0$ if and only if $\operatorname{dim}(Y)>0$, if and only if $y \in Z(V)$.

We define the subset $U$ of points $x \in Y_{n}^{\prime}$ such that if $y:=\pi_{2}(x) \in V$, then there exists a positive dimensional irreducible component of the fiber $\pi_{2}^{-1}(y)$, containing $x$. According to part $(d)$ of 3.22 (page 95) in [Har77], the subset $U$ is Zariski closed. We let $\tilde{Z}:=U \cap \Delta$. Then $\tilde{Z}$ is Zariski closed, and we claim that

$$
\tilde{Z}=\{(y, y) \mid y \in Z(V)\} .
$$

Indeed, if $y \in Z(V)$, then there exists a positive dimensional algebraic $\phi$-module $Y$ such that $(y+Y) \subset V$. We may assume $Y$ is a maximal algebraic $\phi$-module with the property that its translate by $y$ lies in $V$. Then $(y+Y) \times\{y\}$ is an irreducible component of the fiber $\pi_{2}^{-1}(y)$, which contains $(y, y)$ (as shown in Claim 3.6). Therefore, $(y, y) \in U \cap \Delta=\tilde{Z}$. Now, conversely, if $(y, y) \in U$, then there exists a positive dimensional irreducible component $Y^{\prime}$ of $\pi_{2}^{-1}(y)$ passing through $(y, y)$. Then $Y^{\prime}=y+Y$ for some positive dimensional algebraic $\phi$-module $Y$ (see Claim 3.6). Thus $y \in Z(V)$, as desired.

Because $\tilde{Z}$ is Zariski closed, then (3.6.3) yields that also $Z(V)$ is a closed subvariety. This concludes the proof of Theorem 2.4.

Before proceeding to the proof of Theorem 2.5, we will prove several preliminary results.

Lemma 3.7. Let $Y \subset \mathbb{G}_{a}^{g}$ be an algebraic $\phi$-submodule. Then $\phi_{\text {tor }} \cap Y$ is Zariski dense in $Y$.

Proof. Let $m:=\operatorname{dim}(Y)$. Then there exists a suitable finite-to-one, dominant projection $\pi$ of $Y$ on $m$ coordinates of $\mathbb{G}_{a}^{g}$. At the expense of relabelling the coordinates, we may assume the projection is on the first $m$ coordinates. Because $\pi(Y)$ is actually an algebraic group, and $\pi$ is a group homomorphism, we conclude that $\pi(Y)=\mathbb{G}_{a}^{m}$. Moreover, $\pi$ has finite fibers. By abuse of notation, we also denote by $\phi$ the induced action of $\left(\phi_{1}, \ldots, \phi_{m}\right)$ on $\mathbb{G}_{a}^{m}$.

Claim 3.8. The preimage of a torsion point of $\mathbb{G}_{a}^{m}$ through $\pi^{-1}$ is a finite set of torsion points in $Y$. 
Proof of Claim 3.8. Let $x$ be a torsion point of $\mathbb{G}_{a}^{m}$. Let $S_{0}$ be the orbit of $x$ under the action of $\phi$; hence $S_{0}$ is a finite $\phi$-submodule of $\mathbb{G}_{a}^{m}$. Moreover $S_{0}$ is a finite set of torsion points. Because $\pi$ has finite fibers, $S:=\pi^{-1}\left(S_{0}\right)$ is a finite subset of $Y$. Moreover, because $\pi$ commutes with the $\phi$-action, we conclude that $S$ is also a $\phi$-module. Hence, $S$ consists of finitely many torsion points (if $S$ would contain a nontorsion point $z$, then the infinite $\phi$-orbit of $z$ would be contained in $S$, contradicting the fact that $S$ is finite). Therefore, the preimage of $x$ is indeed a finite set of torsion points in $Y$.

Because $\phi_{\text {tor }}\left(\mathbb{G}_{a}^{m}\right)$ is a cartesian product of infinite subsets of the affine line, then $\phi_{\text {tor }}\left(\mathbb{G}_{a}^{m}\right)$ is Zariski dense in $\mathbb{G}_{a}^{m}$. We conclude that the Zariski closure of $\pi^{-1}\left(\phi_{\text {tor }}\left(\mathbb{G}_{a}^{m}\right)\right) \subset Y$ has dimension $m$. Hence, it equals $Y$ (because $Y$ is irreducible). Thus $\pi^{-1}\left(\phi_{\text {tor }}\left(\mathbb{G}_{a}^{m}\right)\right)$ is a Zariski dense set of torsion points in $Y$ (see Claim 3.8). This concludes the proof of Lemma 3.7.

The following key result is an immediate corollary of Lemma 3.7.

Corollary 3.9. There are no infinite algebraic families of algebraic $\phi$-submodules of $\mathbb{G}_{a}^{g}$.

Proof. Using Lemma 3.7, every algebraic $\phi$-submodule of $\mathbb{G}_{a}^{g}$ contains a Zariski dense set of torsion points. Hence each algebraic $\phi$-submodule of $\mathbb{G}_{a}^{g}$ is defined over $K^{\text {sep }}$ (because every torsion point of $\phi$ is defined over $K^{\text {sep }}$ ). Therefore, there are no infinite algebraic families of algebraic $\phi$-submodules of $\mathbb{G}_{a}^{g}$.

Definition 3.10. For an irreducible subvariety $V \subset \mathbb{G}_{a}^{g}$, we call the $\phi$-stabilizer of $V$ (denoted by $\operatorname{Stab}_{\phi}(V)$ ) the largest algebraic $\phi$-submodule $Y$ such that $Y+V=V$.

The $\phi$-stabilizer of $V$ is well-defined because if the algebraic $\phi$-modules $Y_{1}$ and $Y_{2}$ have the property that $Y_{1}+V=V$ and $Y_{2}+V=V$, then the connected component $Y_{0}$ of $\left(Y_{1}+Y_{2}\right)$ is also an algebraic $\phi$-module such that $Y_{0}+V=V$. Moreover, $Y_{1}$ and $Y_{2}$ are contained in $Y_{0}$.

The following result is a corollary of Theorem 2.4.

Corollary 3.11. Let $V \subset \mathbb{G}_{a}^{g}$ be a positive dimensional irreducible affine variety. If $Z(V)=V$, then $\operatorname{dim} \operatorname{Stab}_{\phi}(V)>0$. More precisely, $\operatorname{Stab}_{\phi}(V)$ is the unique maximal algebraic $\phi$-submodule whose translate lies in $V$.

Proof. Using the notation as in the proof of Theorem 2.4, the fact that $Z(V)=V$ yields that $\tilde{Z}=\Delta$. In particular, $\Delta \subset U$. Because $V$ is irreducible, then $\Delta$ is irreducible. Let $U_{0}$ be an irreducible component of $U$ which contains $\Delta$. Then the restriction of $\pi_{2}: Y_{n}^{\prime} \rightarrow V$ to $U_{0}$ is a dominant morphism. By abuse of notation, this restriction will also be called $\pi_{2}$.

The fibers of $\pi_{2}: U_{0} \rightarrow V$ form an algebraic family of algebraic $\phi$-submodules (there is only one family because both $V$ and $U_{0}$ are irreducible). Thus, they are translates of the same positive dimensional algebraic $\phi$-submodule $Y$, since there is no non-constant algebraic family of algebraic $\phi$-modules (see Corollary 3.9). Therefore for each $y \in V$, we have $y+Y \subset V$. Hence $Y \subset \operatorname{Stab}_{\phi}(V)$, which shows that $\operatorname{Stab}_{\phi}(V)$ is positive dimensional. Moreover, no algebraic $\phi$-submodule $Y^{\prime}$ larger than $\operatorname{Stab}_{\phi}(V)$ has a translate which lies in $V$ (because all fibers of $\pi_{2}$ restricted to each irreducible component of $U$ which contains $\Delta$ are translates of the same algebraic $\phi$-module). 
We are ready to prove Theorem 2.5.

Proof of Theorem 2.5. Clearly, there is only one algebraic $\phi$-module of dimension 0. So, let $Y$ be a maximal algebraic $\phi$-module (of positive dimension) whose coset lies in $V$. Therefore, a coset $(y+Y)$ lies in $Z(V)$. Because $Z(V)$ is a closed subset of $V$ (as shown by Theorem 2.4), then $(y+Y)$ lies in one of the finitely many irreducible components $V_{1}$ of $Z(V)$. Because $V_{1}$ is irreducible and $Z\left(V_{1}\right)=V_{1}$ (because $Z(Z(V))=Z(V))$, then Corollary 3.11 shows that there exists a unique maximal algebraic $\phi$-submodule whose coset lies in $V_{1}$. Therefore $Y$ is one of the finitely many $\phi$-stabilizers of the irreducible components of $Z(V)$, which concludes the proof of Theorem 2.5.

\section{Acknowledgements}

I thank Laurent Denis and Thomas Scanlon for a couple of interesting conversations.

\section{References}

[Abr94] D. Abramovich, Subvarieties of semiabelian varieties, Compositio Math. 90 (1994), no. 1, $37-52$.

[Bog81] F. Bogomolov, Points of finite order on an abelian variety, Math. USSR-Izv 17 (1981), no. $1,55-72$.

[Den92] L. Denis, Géométrie diophantienne sur les modules de Drinfel' $d$, The arithmetic of function fields (Columbus, OH, 1991), Ohio State Univ. Math. Res. Inst. Publ., vol. 2, de Gruyter, Berlin, 1992, pp. 285-302.

[Fal94] G. Faltings, The general case of S. Lang's conjecture, Barsotti Symposium in Algebraic Geometry (Abano Terme, 1991), Perspect. Math., no. 15, Academic Press, San Diego, CA, 1994, pp. $175-182$.

[Ghi05] D. Ghioca, The Mordell-Lang theorem for Drinfeld modules, Int. Math. Res. Not. (2005), no. $53,3273-3307$.

[Ghi06] _ Towards the full Mordell-Lang conjecture for Drinfeld modules, submitted for publication, 6 pages, 2006.

[Gos96] D. Goss, Basic structures of function field arithmetic, Ergebnisse der Mathematik und ihrer Grenzgebiete (3) [Results in Mathematics and Related Areas (3)], vol. 35, Springer-Verlag, Berlin, 1996.

[GT07] D. Ghioca and T. J. Tucker, A dynamical version of the Mordell-Lang conjecture for the additive group, to appear in Compositio Math., 14 pages, 2007.

[Har77] R. Hartshorne, Algebraic geometry, Springer-Verlag, New York, 1977.

[Kaw80] Y. Kawamata, On Bloch's conjecture, Invent. Math. 57 (1980), no. 1, 97-100.

Dragos Ghioca, Department of Mathematics and Computer Science, University of LethBridge, 4401 University Drive, Lethbridge, Alberta T1K 3M4, Canada,

E-mail address: dragos.ghioca@uleth.ca 